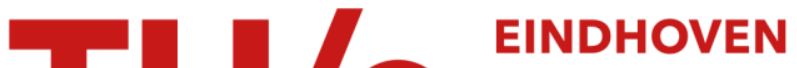 UNIVERSITY OF TECHNOLOGY
}

\section{Motional Stark Effect Diagnostics for KSTAR}

Citation for published version (APA):

Chung, J., Ko, J., Howard, J., Michael, C., Nessi, von, G., Thorman, A., \& Bock, de, M. F. M. (2014). Motional Stark Effect Diagnostics for KSTAR. Journal of the Korean Physical Society, 65(8), 1257-1260. https://doi.org/10.3938/jkps.65.1257

DOI:

10.3938/jkps.65.1257

Document status and date:

Published: 01/01/2014

\section{Document Version:}

Publisher's PDF, also known as Version of Record (includes final page, issue and volume numbers)

\section{Please check the document version of this publication:}

- A submitted manuscript is the version of the article upon submission and before peer-review. There can be important differences between the submitted version and the official published version of record. People interested in the research are advised to contact the author for the final version of the publication, or visit the $\mathrm{DOI}$ to the publisher's website.

- The final author version and the galley proof are versions of the publication after peer review.

- The final published version features the final layout of the paper including the volume, issue and page numbers.

Link to publication

\section{General rights}

Copyright and moral rights for the publications made accessible in the public portal are retained by the authors and/or other copyright owners and it is a condition of accessing publications that users recognise and abide by the legal requirements associated with these rights.

- Users may download and print one copy of any publication from the public portal for the purpose of private study or research.

- You may not further distribute the material or use it for any profit-making activity or commercial gain

- You may freely distribute the URL identifying the publication in the public portal.

If the publication is distributed under the terms of Article 25fa of the Dutch Copyright Act, indicated by the "Taverne" license above, please follow below link for the End User Agreement:

www.tue.nl/taverne

Take down policy

If you believe that this document breaches copyright please contact us at:

openaccess@tue.nl

providing details and we will investigate your claim. 


\title{
Motional Stark Effect Diagnostics for KSTAR
}

\author{
J. Chung and J. Ko* \\ National Fusion Research Institute, Daejeon 305-333, Korea \\ J. Howard, C. Michael, G. von Nessi and A. Thorman \\ The Australian National University, Canberra, Australia \\ M. F. M. De Bock \\ Eindhoven University of Technology, The Netherlands
}

(Received 5 November 2013, in final form 22 February 2014)

\begin{abstract}
The motional Stark effect (MSE) diagnostic is used to measure the radial magnetic pitch-angle profile in neutral-beam-heated plasmas. The diagnostic relies upon the measurement of the polarization direction of Stark-split D-alpha emission from injected fast neutral atoms in a magnetic field. Measurements of the magnetic pitch angle are used with magnetic equilibrium reconstruction codes such as EFIT to calculate the safety factor in shaped plasmas. The MSE diagnostic is important for determining the shape of the q profile to optimize confinement and stability, and it has become a key element in high-performance tokamaks. For the purpose of achieving the high-performance operating region in the Korea Superconducting Tokamak Advanced Research KSTAR device, two types of methods are being studied. In KSTAR, a multichord PEM (photo-elastic modulator)-based MSE system is being developed, and an imaging MSE polarimetry system using the coherence imaging technique has been showing promising initial results during the last two KSTAR experimental campaigns in 2012 and 2013, respectively. In this paper, we describe the progress of the KSTAR MSE diagnostics.
\end{abstract}

PACS numbers: 52.70.Kz, 52.70.-m, 52.55.Fa

Keywords: MSE, Motional Stark effect, q-profile, KSTAR

DOI: $10.3938 /$ jkps.65.1257

\section{INTRODUCTION}

The MSE diagnostic is used to measure the radial magnetic pitch-angle profile in neutral-beam-heated plasmas. The diagnostic relies upon the measurement of the polarization direction of the Stark split D-alpha emission from injected fast neutral atoms in a magnetic field. The injected neutral atom with velocity $v$ in a magnetic field $B$ experiences a Lorentz electric field $E=v \times B$, which causes a Stark splitting into orthogonally-polarized components, $\sigma$ and $\pi$, of the emitted D-alpha line. The Stark $\sigma$ and $\pi$ components are polarized perpendicular and parallel to the direction of the electric field, respectively. Measurement of the magnetic pitch angle is used, with magnetic equilibrium reconstruction codes such as EFIT, to calculate the safety factor, $q$, in shaped plasmas. The MSE diagnostic is important for shaping the q profile to optimize the confinement and the stability, and it has become a key element in high-performance tokamaks. In the KSTAR, two types of methods are

*E-mail: jinil@nfri.re.kr; Fax: +82-2-554-1643 being studied under strong international collaborations with the Australian National University (ANU) and the Eindhoven University of Technology (TU/e).

An imaging MSE polarimetry system using the coherence imaging technique has shown promising initial results during the last two KSTAR experimental campaigns in 2012 and 2013, respectively. The spectropolarimetric optical system developed by ANU allows 2dimensional (2D) imaging of the vertical magnetic field in a tokamak plasma [1-3]. The system provided the first internal magnetic data for constraining the KSTAR equilibrium.

A conventional PEM (photo-elastic modulator)-based multichord MSE system is also being developed for realtime plasma-current-profile control, and this will be commissioned in two years. Collection optics relay the beam emission through two crossed photo-elastic modulators (dual PEM). The dual PEM modulates the direction of the polarization at two frequencies. A narrow-band interference filter selects the light from only one of the $\sigma$ and the $\pi$ components after which it is detected. The ratio of the detector signal at twice the modulating fre- 


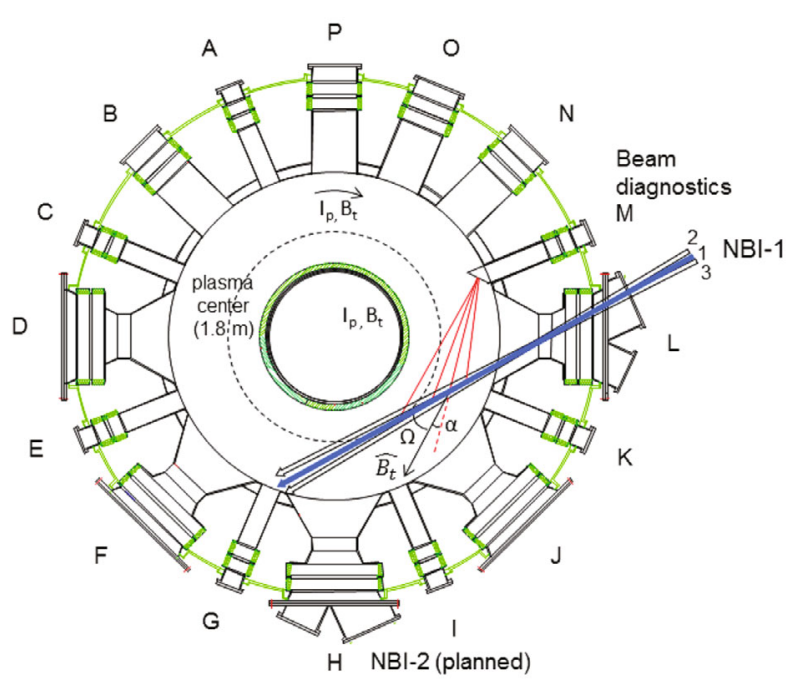

Fig. 1. (Color online) Top view of the KSTAR showing locations of a set of neutral beams from the NBI-1 on port L and of the beam diagnostic cassette on port $\mathrm{M}$.

quencies is the proportional to the magnetic pitch angle. The grating survey spectrometer is used for the system calibration, and a digitizer with the embedded digital lock-ins will be utilized for effective data processing for real-time control.

Section II describes the MSE viewing geometry and the specifications of the NBI system for a beam diagnostic. Section III contains the recent progress of the $2 \mathrm{D}$ MSE using the coherence imaging approach. Section IV discusses the development of a conventional multichord MSE. The summary is found in Section V.

\section{VIEWING GEOMETRY AND THE NEUTRAL BEAM}

The KSTAR MSE observes the Stark splitting of the D-alpha line at $656.1 \mathrm{~nm}$ by the electric field associated with neutral deuterium atoms injected from the first neutral heating beam system called NBI-1. Figure 1 shows the location of a cassette of the beam diagnostic and the neutral beam. The KSTAR has a maximum toroidal field of $3.5 \mathrm{~T}$ at a major radius of $1.8 \mathrm{~m}$, a minor radius of $0.5 \mathrm{~m}$, and an operational plasma current of up to 1 MA (2013 campaign). Directions of both the toroidal field and the plasma current are clockwise. The radial resolution is given by $\delta R=(d \sin \alpha+w \sin \Omega) / \sin (\Omega+$ $\alpha$ ), where $d$ is the optical spot size, $w$ is the beam width, and the angles $\alpha$ and $\Omega$ are defined with a projection of an observation line, the beam, and the magnetic field as indicated in the Fig. 1 [4]. The tangential view of the NBI-1 heating beam provides a good spatial resolution of $2-3 \mathrm{~cm}$. This gives an opportunity to have a multichord system with a maximum of 25 channels to cover the major radius from 1.7 to $2.3 \mathrm{~m}$.

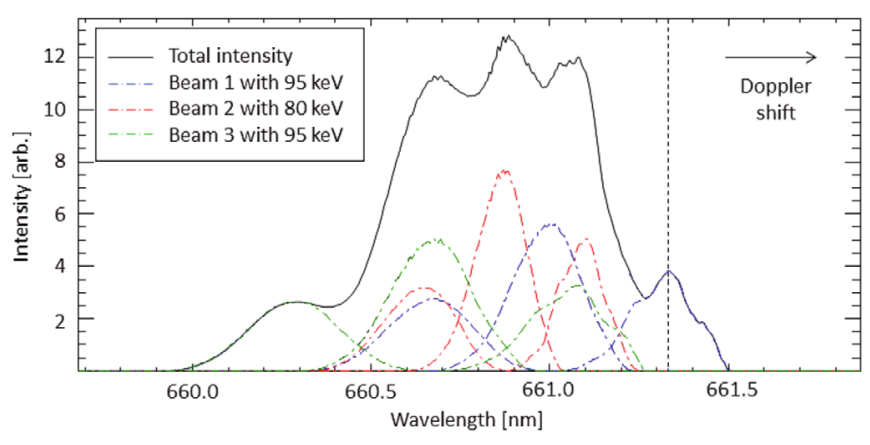

Fig. 2. (Color online) Simulation of a full-energy spectrum for a 95-80-95 keV deuterium beam configuration of the NBI1. An isolated $\pi$ component of beam 1 (marked with a dashed vertical line) may be a candidate of the measurement.

The NBI-1, which lies in the horizontal mid plane, consists of three positive ion sources, and it was designed to accept a beam with a divergence angle of less than 1 degree (or $<0.17 \mathrm{rad}$ ). The shape of a cross section of each beam is a vertically-elongated rectangle $(0.2 \times 0.6$ $\left.\mathrm{m}^{2}\right)$. The first ion source launched in 2010 delivered a beam power of $1.5 \mathrm{MW}$ at a beam energy of $95 \mathrm{keV}$ [5]. The second ion source, which has been available from the 2013 campaign, has a beam power of $2.0 \mathrm{MW}$ at a $95-\mathrm{keV}$ beam energy while the third, which is the last one in the NBI- 1 , is expected in 2014 with the same performance as the second one. The injection angles of the second and the third beams relative to the center of the first (middle) beam are 4 degrees each. This is a bad condition for the MSE measurement because the spectrum of the Stark splitting is much more complicated, and the beam width is broader when all three beams are injected together. However, modulating the beam is a solution for a reference measurement. Additionally, adjusting the energy of each beam (in the range of $80-$ $95 \mathrm{kV}$ ) can result in an isolated line at the edge of the total Stark spectrum due to the Doppler shift. Figure 2 shows the initial result of an atomic simulation for designing a narrow band-pass filter for a multichord MSE system. The $\pi$ component of beam 1 can be seen not to be contaminated by the other beams.

A 2-m-long cassette system for the beam diagnostic has been installed with a tangential view of the tokamak on the median port $M$ with the NBI-1. The tangential view gives an advantage that no in-vessel optical array will be required. The cassette has three viewport windows vertically, and it was designed to contain a $2 \mathrm{D}$ MSE, a multichord MSE, a CES (charge-exchange recombination Spectroscopy), and a BES (Beam Emission Spectroscopy) system from the top window. Here the 2D MSE system is installed $28.5 \mathrm{~cm}$ above the mid plane while the multichord MSE and CES system share the window in the mid plane. The windows are protected by a remotely-operated in-vacuum shutter from maintenance operations such as wall-cleaning discharges and boronizations. 


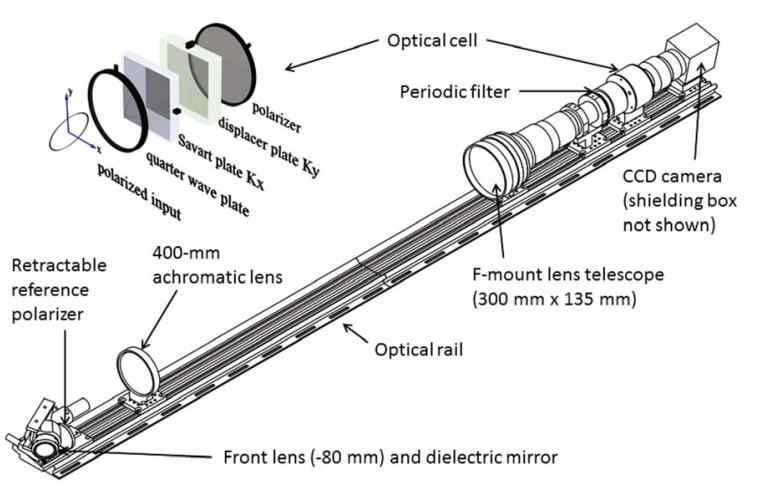

Fig. 3. Optical layout of the 2D MSE system on KSTAR M-port.

\section{2D IMAGING MSE POLARIMETRY}

The imaging MSE polarity is a new approach to measuring the internal current profile. This is a compact 2D MSE system that delivers the spatial distribution of the vertical magnetic field in the region of the plasma illuminated by the neutral heating beam [6]. A proof-ofprinciple study of the 2D MSE system was carried out in the 2010 and the 2011 campaigns on the top window of the M-port in the KSTAR, and the first successful system was installed and operated in 2012 and 2013 as shown. The system uses a switching polarimeter to produce an image of the beam with a superimposed interference fringe pattern. The phase difference between adjacent frames gives the polarization orientation angle. Because the fringe phase is parallel with the radial direction, the radial spatial resolution is approximately limited by the line-of-sight integration through the beam and is on the order a few mm. This is a significant advantage for imaging the edge pedestal region.

Figure 3 shows a schematic of the system. Because the viewing window is $28.5 \mathrm{~cm}$ above the mid plane, the front lens is tilted 7.5 degrees to look down on the beam. The dielectric mirror reflects the incoming light while keeping its polarization state, and the achromatic lens relays the light to the focusing telescope lens. Finally, the neutral-beam's image is focused onto an imaging CCD after passing an optical cell consisting a Savart plate, a displacer, and an analyzing polarizer. The optical system setup was refined after the first two campaigns and features telephoto lenses to bring the light $\sim 1.5 \mathrm{~m}$ out so that the optics and the camera can be situated outside the cryostat to reduce the influence of neutrons/gamma rays and to enable easy reconfiguration of the optics. The raw data and the EFIT fit, together with the inferred q profile, are shown in Figs. 4(a) and (b). The raw polarization angle map and the EFIT fit based only on those 18 mid plane points are shown in Figs. 4(c) and (d). The high degree of correspondence at locations off mid plane provides evidence that vertical variations in the polarization image are not inconsistent with a Grad- (a) Midplane Polarization Angle Profiles

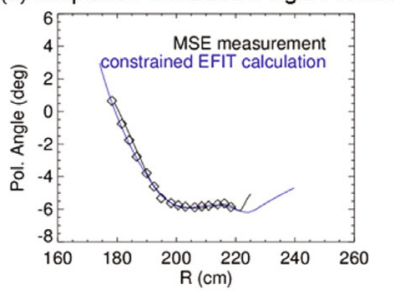

(c) Measured Angle
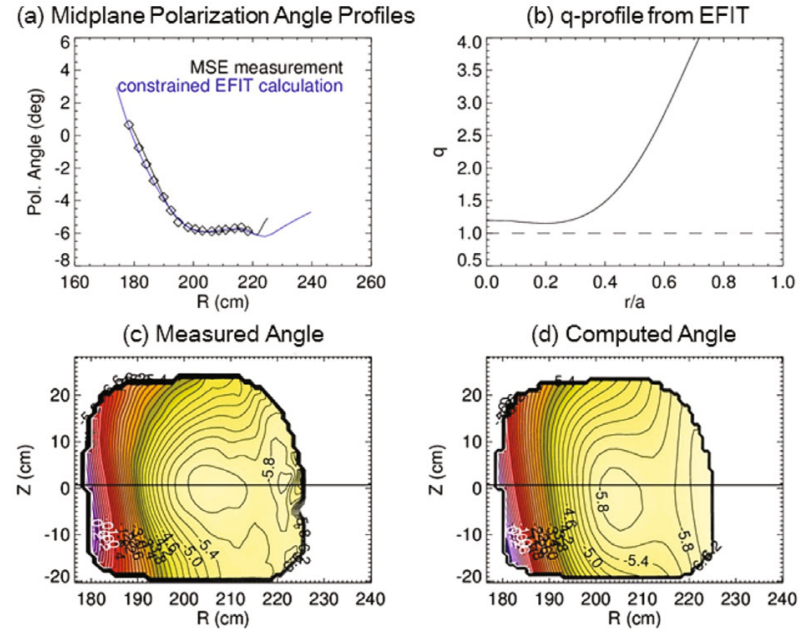

(d) Computed Angle

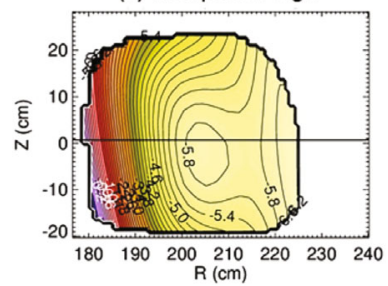

Fig. 4. (Color online) Results of the 2D MSE measurements: (a) mid plane polarization angle profile together with best fit EFIT, (b) EFIT $q$ profile, (c) measured 2D polarization image, and (d) calculated image based on the EFIT solution constrained only by 18 mid plane points.

Shafranov-based equilibrium.

\section{DEVELOPMENT OF A MULTICHORD MSE FOR REAL-TIME CONTROL}

A PEM-based polarization measurement of a single Stark line with a narrow band-pass filter is a standard method in major tokamaks such as the JET, ASDEX-U, DIII-D, JT-60 and MAST [7-10] and will be used for the multichord MSE system.

The KSTAR MSE system may be used for real-time current-profile control. While the PEM-based technique is conventional, its application to real-time control is a challenge for the steady-state operation in tokamaks. Strategies for steady-state high-performance advanced tokamak scenarios are highly related to control of the current density [11]. Application of real-time control is a long-term physical and technical goal after the commissioning in the KSTAR which is expected in 2015.

Figure 5 shows a conceptual layout of the components of the multichord MSE system in the KSTAR. Because the MSE system needs to share a vacuum window with the CES system on the mid plane in the cassette, a collection optic system is being designed for both diagnostics by using a dichroic beam splitter that splits the incoming light into two wavelength ranges. This will help to avoid attenuation of the light intensity for the CES system at the linear polarizer.

A dual PEM, two PEMs mounted in a single enclosure, is a key optical component in the polarization measurement. Optical retardation axes of the two PEMs are at 45 degrees with respect to each other, with one operating at $20 \mathrm{kHz}$ and the other at $23 \mathrm{kHz}$. This is the setup 


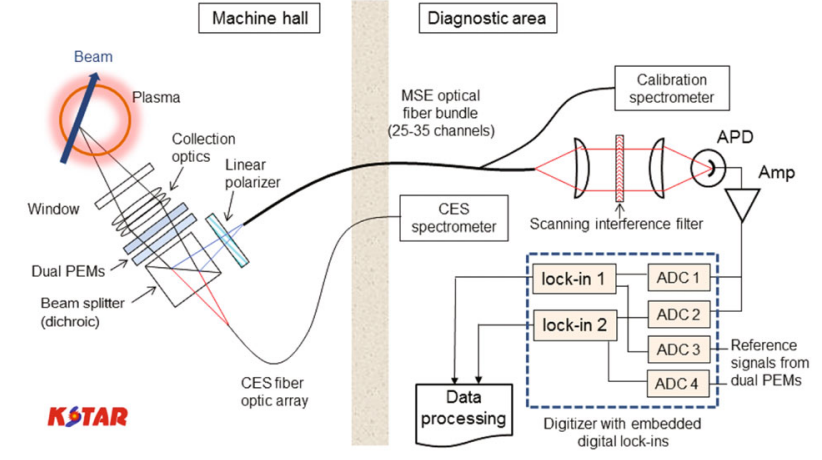

Fig. 5. (Color online) Optical arrangement of the PEMbased multichord MSE system in the KSTAR. The MSE and the CES system share their collection optics before a dichroic beam splitter. A digital lock-in technique will help real-time $q$-shaping control to optimize the confinement and the stability.

for a Stokes polarimeter with the linear polarizer passing axis at 22.5 degrees relative to each PEM. This modulates the polarization state, represented by four Stokes parameters, of the incoming light into the intensity and the polarization fraction. Therefore, demodulating the output signal allows us to derivate the polarization state of the incoming light. The housing of a dual PEM for the KSTAR MSE system is made of a plastic material, which is non-magnetic and non-conductive, to survive in a high superconducting TF magnetic field that stays on all the time during the experiment. The opening of the optical surface is $100 \mathrm{~mm}$, but about $80 \%$ of it might be useful because of deviations of the retardance over the surface. According to a report on test measurements of the KSTAR dual PEM, the retardance for both PEMs varies over the surface of the aperture by up to $50 \%$ from the center to the edge, and the ratio of retardances of the PEMs is best (1.0) for an aperture of $5 \mathrm{~cm}$ in diameter [12]. This was double-checked recently, and the information is being used as an important input parameter for the design of the collection optics.

The candidate Stark line is isolated by using scanning multi-cavity narrowband filters mounted in the diagnostic area. A special digital signal processing (DSP) firmware allows a digitizer to function as a digital lockin amplifier. Fifty-four data acquisition channels with a maximum sampling rate of $>1 \mathrm{MHz}$ will be used. Twenty-five channels are for a real-time DSP, 4 channels are for reference signals from the PEMs, and the last 25 channels are used as a second shadow digitizer that records the full raw data for verification and possible additional post-shot lock-in processing.

\section{SUMMARY}

There are two challenges for measuring pitch angles with the MSE in the KSTAR. The first is spectral over- lap of each of the three ion sources in NBI-1, which gives a complexity to the Stark spectrum. Strategies to overcome this are being investigated based on simulations of the Stark components cross-checked with spectral measurement. Also, the space available for the optical components in the beam diagnostic cassette is very limited, and the window on the mid plane has to be shared between CES and MSE. Various options are being considered to overcome this. An imaging MSE polarimetry system using the coherence imaging technique has shown promising initial results in the KSTAR for the last couple of years. A conventional PEM-based multichord MSE system, which will be commissioned in two years, is also being developed. The real-time control of the current profile is a long-term challenge for both systems.

\section{ACKNOWLEDGMENTS}

The authors would like to thank R. Huijgen, D. Aussems, H. Ong, and G. Lange for helpful discussions and useful technical reports for the KSTAR multichord MSE development during their internship at the National Fusion Research Institute. This work was supported by the Korean Ministry of Science, ICT and Future Planning under the KSTAR project contract. Part of this work was also undertaken with funding support from the Australian Research Discovery Program under Grant DP110104833.

\section{REFERENCES}

[1] J. Howard, Plasma Phys. Control. Fusion 50, 125003 (2008).

[2] J. Howard and Jinil Chung, Rev. Sci. Instrum. 83, 10D510 (2012)

[3] A. Thorman, C. Michael, J. Howard, Rev. Sci. Instrum. 6, 063507 (2013)

[4] B. W. Rice, D. G. Nilson and D. Wróblewski, Rev. Sci. Instrum. 66, 373 (1995).

[5] S. H. Jeong et al., Rev. Sci. Instrum. 83, $02 B 102$ (2012).

[6] J. Howard, A. Diallo, R. Jaspers and J. Chung, J. Plasma Fusion Res. 5, S1010-1 (2010).

[7] N. C. Hawkes, K. Blackler, B. Viaccoz, C. H. Wilson, J. B. Migozzi and B. C. Stratton, Rev. Sci. Instrum. 70, 894 (1999) .

[8] B. W. Rice, K. H. Burrell and L. L. Lao, Nuclear Fusion 37, 517 (1997).

[9] T. Fujita, H. Kuko, T. Sugie, N. Isei and K. Ushigusa, Fusion Engin. Design 34-35, 289 (1997).

[10] N. J. Conway et al., Rev. Sci. Instrum. 81, $10 \mathrm{D} 738$ (2010).

[11] D. Moreau et al., Nucl. Fusion 43, 870 (2003).

[12] Technical report, Ralph T. Huijgen, The Eindhoven University of Technology (2011). 